\title{
27 SARS CoV and other coronaviruses
}

\section{The viruses}

Coronaviruses $(\mathrm{CoV})$, including SARS $\mathrm{CoV}$, are single-stranded RNA viruses and belong to the family Coronaviridae.

\section{Epidemiology}

\section{Route of spread}

Coronaviruses are spread by the respiratory route.

Severe acute respiratory syndrome (SARS) is caused by SARS coronavirus (SARS $\mathrm{CoV}$ ), which is spread by the respiratory route and through the ingestion of aerosolized faeces via contamination of the hands and environment. Close contact with a symptomatic person poses the highest risk of infection. In the 2003 outbreak, most cases occurred in hospital workers or family members in contact with cases.

\section{Prevalence}

Coronaviruses have a worldwide distribution and almost all adults in the UK have been infected by at least one type of coronavirus. Infection usually occurs in winter or spring and is associated with upper respiratory tract infection (a 'cold'). The severity of illness is similar to that of rhinovirus infection, but less severe than infection with respiratory syncitial virus or influenza viruses. Symptoms are usually more severe in elderly persons. Reinfection is common.

SARS CoV caused a worldwide outbreak between March and July 2003, and there was a smaller outbreak, probably associated with laboratory-released SARS CoV, in 2004. There were over 8000 cases reported from 32 countries. There have been no more cases since then. The outbreak originated in Guandong Province in China and is thought to have been transmitted from civet cats (a variety of wild cat) to humans with subsequent human-to-human spread.

\section{Incubation period}

2-4 days (SARS has an incubation period of 2-7 days).

\section{Infectious period}

Coronaviruses are considered infectious while patients are symptomatic. Patients are most infectious at the onset of respiratory symptoms. 


\section{At-risk groups}

Coronavirus infection is more severe in elderly persons. The most severe and fatal infections with SARS have been in elderly persons.

\section{Clinical}

\section{Symptoms}

Coronaviruses produce a range of symptoms from asymptomatic infection to upper respiratory symptoms (a 'cold'), malaise and fever. Brochiolitis and other lower respiratory tract symptoms occur in a few patients of all ages.

Coronaviruses can be found in the human gut, but there is no clear disease association.

SARS CoV causes high fever $\left(>38^{\circ} \mathrm{C}\right)$, dry cough, shortness of breath, myalgia, headache and diarrhoea. Chest X-rays show pneumonia or respiratory distress syndrome. Symptoms are usually severe enough to warrant hospital admission. The overall fatality rate was $15 \%$ in previous outbreaks, higher in elderly patients and those with other respiratory conditions; less than $1 \%$ in persons less than 24 years of age, $6 \%$ in those aged $25-44$ years, $15 \%$ in those aged $45-64$ years and greater than $50 \%$ in persons aged 65 years or older.

\section{Differential diagnosis}

Parainfluenza, RSV, adenovirus and other respiratory viral infections have to be considered in the differential diagnosis of respiratory CoV infections.

SARS CoV infection has to be distinguished from influenza and other causes of pneumonia and high fever.

\section{Laboratory diagnosis}

Coronaviruses are not usually tested for in most hospital laboratories, although some regional virology laboratories may include coronavirus detection in their respiratory PCR diagnostic test repertoire. They are difficult to grow in cell culture.

SARS $\mathrm{CoV}$ is only diagnosed in specialist reference laboratories with Category 4 diagnostic facilities. In England there are two centres of the Health Protection Agency, in London and Porton Down, that are able to undertake this work. Infection can be diagnosed by PCR or serological tests.

\section{Management}

\section{Treatment}

There is no specific treatment for coronavirus infections, including SARS CoV. Management of the latter requires supportive management of the presenting symptoms. 


\section{Infection control}

The spread of coronaviruses can be reduced by strict handwashing after patient contact. The use of gloves, face masks, aprons and goggles will reduce the risk of transmission. Isolation in single rooms or cohort nursing reduces the risk to other patients.

Severe acute respiratory syndrome is less infectious than influenza but symptomatic patients should be nursed in side rooms, preferably negative-pressure rooms, with gloves, aprons and a respirator mask conforming to at least European standard EN149:2001 FFP3. If a suitable respirator mask is not immediately available, use a surgical face mask (see latest guidance).

\section{Useful website}

Refer to www.hpa.org.uk for more information on diagnosis and infection control for SARS CoV. 